\title{
Recurrent Pulmonary Embolism and Pulmonary Hypertension in a Patient with $\beta$-Thalassemia Intermedia
}

Antonella Tufano ${ }^{1 *}$, Adriana Gianno ${ }^{1}$, Antonio Coppola ${ }^{1}$, Roberta Esposito², Paolo Conca ${ }^{1}$, Gianmarco Alcidi ${ }^{1}$, Nicoletta Franco ${ }^{1}$, Mariateresa Polimeno', Michele D'Alto ${ }^{3}$, Maurizio Galderisi ${ }^{1}$ and Giovanni Di Minno ${ }^{1}$

${ }^{1}$ Department of Clinical Medicine and Surgery, Regional Reference Centre for Coagulation Disorders, Federico II University Hospital, Naples, Italy ${ }^{2}$ Department of Advanced Biomedical Sciences, Interdepartmental Laboratory of Echocardiography, Federico II University Hospital, Naples, Italy

${ }^{3}$ Department of Cardiology, Second University of Studies, AORN Monaldi, Naples, Italy

\begin{abstract}
Patients with $\beta$-thalassemia intermedia are at increased risk of thromboembolic events and multifactorial pulmonary arterial hypertension. A pro-thrombotic state, including decreased levels of natural anticoagulant proteins and chronic platelet activation, has been shown in these patients, in particular after splenectomy.

We report the case of a 54-year-old splenectomized $\beta$-thalassemic patient with a history of unprovoked deep venous (femoro-politeal) thrombosis, complicated by a pulmonary embolism event at the age of 37 , recurrent episodes of superficial vein thrombosis of the lower limbs with leg ulcers, and a progressive severe pulmonary arterial hypertension, related to recurrences of pulmonary embolism, despite long-term, well conducted oral anticoagulant treatment (vitamin $\mathrm{K}$ antagonists and apixaban). After the performance of a right heart catheterization, pulmonary endarterectomy was not considered indicated in this patient because of the distal localization of thrombi. Aspirin treatment was added to vitamin $\mathrm{K}$ antagonists. Riociguat and ambrisentan therapy induced an improvement of both symptoms and echocardiographic picture over 12-mo follow-up.
\end{abstract}

Splenectomized thalassemic patients are at high risk of thromboembolic events, and pulmonary hypertension, despite common, is yet a poorly understood complication. Clear recommendations on the management of such condition are lacking due to the limited data regarding the use of vasodilators, anticoagulants (vitamin $\mathrm{K}$ antagonists, heparins, direct oral anticoagulants) and antiplatelet agents.

Keywords: Beta-thalassemia; Pulmonary embolism; Pulmonary arterial hypertension; Aspirin; Riociguat; Ambrisentan

\section{Introduction}

Thalassemia is a congenital hemolytic disease caused by defective $\alpha$ - or $\beta$-globin chain synthesis $[1,2] . \beta$-thalassemia intermedia (TI) is characterized by mild to moderate decrease in $\beta$ globulin production, and by a wide clinical spectrum that lies between that of thalassemia minor and major (TM) $[1,2]$. Three main factors lead to the clinical presentation of TI: ineffective erythropoiesis, chronic anemia/hemolysis and iron overload, secondary to increased intestinal absorption [3,4]. Venous thromboembolism (VTE), including pulmonary embolism (PE), deep vein thrombosis (DVT) and portal vein thrombosis, has been reported in adult TI patients [5-8]. A hypercoagulable state has been identified in this setting, attributed to several factors, including pro-coagulant activity of circulating damaged red blood cells (RBCs), high levels of pro-coagulant microparticles, increased platelet activation, decreased levels of anticoagulant Protein C and Protein S, and endothelial injury/activation [7]. These factors have been observed at high rate in splenectomized TI patients $[9,10]$. Both TM and TI are associated with the development of pulmonary hypertension $(\mathrm{PH})$ $[11,12]$, which has been shown at echocardiography in $10-75 \%$ and 40 $50 \%$ of TM and TI patients, respectively [12].

Limited evidence exists for defining the optimal antithrombotic treatment and the use of vasodilators to treat $\mathrm{PH}$ associated with $\mathrm{TI}$ [12]. We here report the case of a splenectomized $\beta$-thalassemic patient with severe $\mathrm{PH}$ due to recurrent $\mathrm{PE}$, despite initial oral anti-coagulation with vitamin $\mathrm{K}$ antagonists (VKAs) and then with apixaban.

\section{Case Report}

A 54-year-old man was admitted to our Department in October 2016 because of progressively worsening dyspnea, even at rest, since few weeks. He was referred for the first time to our tertiary care Department in 1999, at the age of 37, when an apparently unprovoked DVT of the left femoral-popliteal vein, complicated by PE occurred. The patient was affected by $\beta$-TI and underwent splenectomy at the age of 20 years. The thrombophilia workup [including FV Leiden and prothrombin (FII) G20210A gene polymorphisms, plasma anticardiolipin and anti- $\beta$ 2 glycoprotein 1 antibodies, lupus anticoagulant, plasma antithrombin, Protein C and Protein S, and homocysteine] was negative nor clinical investigation allowed to identify further concomitant diseases. The blood assay showed mild anemia $(11.0 \mathrm{~g} / \mathrm{dL})$, leucocytosis $(14.000 /$ $\mathrm{mmc}$ ) and thrombocytosis $(491.000 / \mathrm{mmc})$, related to the TI and the previous splenectomy. After heparin therapy during the acute phase, oral anticoagulant treatment (OAT) with VKA (INR target 2.0-3.0) was started, without pre-defined time duration because of the idiopathic nature of the thromboembolic episode. Despite OAT and INR within the target range, two episodes of superficial vein thrombosis (SVT) of the lower limbs occurred, four and six years after the first thromboembolic event respectively. They were successfully managed with therapeutic doses of low molecular weight heparin (EBPM). However, the patient developed chronic venous insufficiency and leg ulcers. Clinical follow-

*Corresponding author: Antonella Tufano, Department of Clinical Medicine and Surgery, Regional Reference Centre for Coagulation Disorders, Federico II University Hospital, Naples, Italy, Tel: +39 3337394490; E-mail: atufano@unina.it

Received January 30, 2018; Accepted February 08, 2018; Published February 12, 2018

Citation: Tufano A, Gianno A, Coppola A, Esposito R, Conca P, et al. (2018) Recurrent Pulmonary Embolism and Pulmonary Hypertension in a Patient with $\beta$-Thalassemia Intermedia. J Blood Lymph 8: 205. doi:10.4172/2165-7831.1000205

Copyright: $\odot 2018$ Tufano A, et al. This is an open-access article distributed under the terms of the Creative Commons Attribution License, which permits unrestricted use, distribution, and reproduction in any medium, provided the original author and source are credited. 
up was negative for further overt VTE events until October 2016. At that time, a CT-scan showed recurrence of unilateral segmental $\mathrm{PE}$, despite the on-going and well dosed OAT. By a transthoracic echocardiographic exam continuous-wave Doppler highlighted a severe tricuspid valve regurgitation with a retrograde gradient $=88$ $\mathrm{mmHg}$ (estimated pulmonary arterial systolic pressure $[\mathrm{PAPs}]=93$ $\mathrm{mmHg}$ ) whereas right ventricular (RV) internal cavity dimension was increased with the typical D-shaped configuration of severe $\mathrm{PH}$. Treatment with VKA was stopped and, after 1-week enoxaparin therapy (100 IU /Kg body weight twice daily) the direct anti-Xa inhibitor apixaban ( $5 \mathrm{mg}$ twice daily) was started. Nevertheless, two months later the patient was urgently re-admitted to our Department because of worsening dyspnea. By the echo exam a further increase of PAPs (144 $\mathrm{mmHg}$ ) was detected whereas RV function was preserved (TAPSE $=22$ $\mathrm{mm}$ ) and right atrial pressure was in the normal or highly normal range (Figure 1). Lung CT-scan evidenced multiple PE recurrence with bilateral segmental and subsegmental arterial thrombosis. Laboratory and instrumental examinations ruled out other conditions associated with acquired thrombophilia, including connective diseases, cancer and myeloproliferative diseases, and paroxysmal nocturnal hemoglobinuria ( $\mathrm{PNH})$. The thrombophilia workup was repeated and confirmed as negative. The ventilation/perfusion (V/Q) scan demonstrated small segmental defects of both lungs. At right heart catheterization (RHC), mean and diastolic pulmonary arterial pressures were $44 \mathrm{mmHg}$ and $5 \mathrm{mmHg}$ respectively, with a normal pulmonary wedge pressure $(=8 \mathrm{mmHg})$; cardiac index was $3.5 \mathrm{l} /$ $\mathrm{min} / \mathrm{m}^{2}$, and pulmonary vascular resistance $=4.95$ Wood Units. The comprehensive analysis of the cath data allowed therefore to identify a pre-capillary pulmonary hypertension. These findings were consistent with a diagnosis of chronic thrombo-embolic pulmonary hypertension $(\mathrm{CTEPH})$. An inferior vena cava filter was therefore placed while planning a pulmonary endarterectomy. However, the patient was then considered inoperable because of the distal localization of thrombi. He was discharged on combined antithrombotic treatment with VKA (INR target 2-3) plus $100 \mathrm{mg}$ aspirin, diuretics and riociguat. After 6
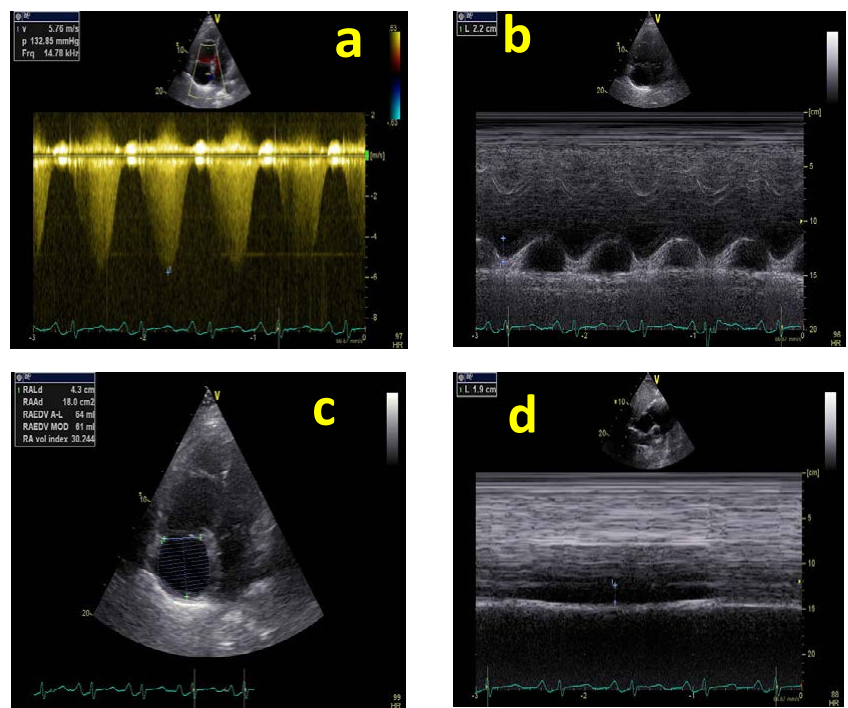

Figure 1: Summarization of the echo exam showing a severe continuous wave Doppler derived tricuspid regurgitation with a retrograde gradient $=132.85$ $\mathrm{mmHg}$ (A), preserved RV function (tricuspid annular plane systolic excursion=22 $\mathrm{mm}$ ) $(B)$, and normal or highly normal estimated RA pressure as derived from a normal RV size $(C)$ and inferior cava vein which is dilated but still keeps a normal respiratory reactivity (D). months, because of the persistence of dyspnea and $\mathrm{PH}$, ambrisentan, 10 mg daily, was added. After six months of such treatment, a significant improvement in functional class, echocardiographic parameters and 6-minute walk test were seen, without significant adverse effects.

\section{Discussion}

The presence of a hypercoagulable state in patients with TI is well recognized [5-8]. Thromboembolic events are more frequent in TI patients than in patients with TM, more venous events occurring in TI and more arterial thrombosis in TM [13]. The hypercoagulable state in patients with $\beta$-TI has been attributed to several factors [7,8]. It is widely accepted that patients with thalassemia have chronically activated platelets, and enhanced platelet aggregation, as confirmed by the increased expression of CD62P (P selectin) and CD63, markers of in vivo platelet activation. The platelet survival is shortened as a consequence of platelet activation and consumption $[14,15]$. Thalassemic patients have also a 4 to 10 fold increase of urinary metabolites of prostacyclin (PGI2) and thromboxane A2 (TXA2), compared to control subjects [16]. Thalassemic deformed RBCs have a pro-coagulant effect, that can result from the expression of negatively charged phospholipids, which facilitate thrombin generation $[7,15]$. Endothelial injury/activation and high levels of pro-coagulant microparticles (of RBC, leucocytic and endothelial origin), also have a pathogenetic contribution in the hypercoagulability of thalassemic patients [7]. Inherited thrombophilia does not play a relevant role in this setting; however, low protein $\mathrm{C}$ and $S$ levels have been showed $[7,17,18]$. The presence of cardiac, hepatic, or endocrine dysfunction in case of severe iron overload may also contribute to such a hypercoagulable state $[17,18]$. Splenectomy contributes to the increased susceptibility to thromboembolism: splenectomized thalassemic patients have high platelet counts and increased number of abnormal RBCs [7,9].

$\mathrm{PH}$ is frequent among patients with TI and TM (rate of about 40$50 \%$ ), and is multifactorial $[11,12]$. Pathogenic mechanisms include:

1. Chronic hemolysis, with decreased nitric oxide bioavailability and platelet activation, increased expression of adhesion molecules and increase of endotelin-1;

2. Iron overload due to transfusions, that leads to fibrosis and dysfunction of the heart;

3. Hypercoagulability and increased incidence of VTE;

4. Changes in circulating cells after splenectomy [12].

The higher occurrence of $\mathrm{PH}$ in splenectomized patients who develop VTE may suggest a common underlying etiology between the two complications [12].

PH is commonly arranged into five separate groups (based on common manifestations, clinical presentation, and therapeutic strategies): 1) Pulmonary arterial hypertension (PAH), 2) $\mathrm{PH}$ due to left heart disease, 3) $\mathrm{PH}$ due to lung diseases and/or hypoxia, 4) Chronic thromboembolic pulmonary hypertension (CTEPH) and other pulmonary artery obstructions, and 5) $\mathrm{PH}$ with unclear and/or multifactorial mechanisms [19]. PH due to chronic haemolytic anaemia is included in group 5, because of its multifactorial mechanisms [12,1922].

Echocardiography can identify thalassemic patients at risk for $\mathrm{PH}$ in whom tricuspid regurgitation velocity is used to estimate RV systolic pressure but is not sufficient to support a treatment and RHC is required [22,23]. In our case, the presence of CTEPH was established 
by RHC, but the patient was classified inoperable because of the distal thrombosis localization at CT scan.

Recent guidelines for the treatment of $\mathrm{PH}$ do not provide specific recommendations for these patients [22], and the literature lacks proper evidence on the role of vasodilators, anticoagulants and antiplatelet agents in the management of $\mathrm{PH}$ in thalassemia. A chronic transfusion therapy, in addition to iron-chelation, is thought to prevent and ameliorate $\mathrm{PH}$ in these patients [12,24,25].

In particular, data regarding the use of pulmonary vasodilators in thalassemic patients are limited and no randomized trials are available to support the routine use of these treatments [12]. Studies using sildenafil, a phosphodiesterase type 5 inhibitor (PDE-5 inhibitor), or endothelin receptor antagonists (ERAs), particularly bosentan, in patients with $\mathrm{PH}$ as a result of sickle cell disease are not conclusive [12].

Randomized, placebo-controlled trials in both PAH and CTEPH patients have shown improvements in WHO functional classification, 6-minute walk distance, and invasive pulmonary hemodynamics with the use of the soluble guanylate cyclase (sGC) stimulator riociguat $[26,27]$. Soluble guanylate cyclase converts guanosine triphosphate (GTP) to cGMP, leading to vasorelaxation. However, published trials did not include patients with $\mathrm{PH}$ related to chronic haemolytic anaemia.

Improvements in both functional class and invasive hemodynamic measurements by RHC are reported in TM complicated by $\mathrm{PH}$ after treatment with epoprostenol, a synthetic prostacyclin [28]. A more recent case report describes a patient with TI and severe $\mathrm{PH}$ diagnosed on RHC who was successfully treated with epoprostenol for more than 3 years with improvements in symptoms, functional classification, 6-minute walk distance, and invasive hemodynamic measurements [21].

Although platelet activation is considered a pathogenic mechanism of PH in TI, data from the literature suggest that aspirin may reduce the risk of recurrence in patients with a history of thromboembolism; however the difference is not statistically significant [13]. In a recent retrospective study on 63 thalassemic patients with $\mathrm{PH}$, the pulmonary artery systolic pressure was unchanged after 1-year treatment with lowdose aspirin [28,29].

We have here reported a patient with $\beta$-TI with recurrent pulmonary embolism complicated by $\mathrm{PH}$, who had clinical and imaging improvements with the association of OAT, aspirin, riociguat and ambrisentan, even in the absence of specific recommendations on the use of these last three drugs.

Therefore, current literature evidence do not allow to draw agreed, standardized approaches for the management of these patients. Further well-designed studies, recruiting sufficient numbers of patients are needed to determine the optimal treatment for this severe condition, often life-threatening, in particular to elucidate the role of aspirin and of the different vasodilator agents.

\section{Authorship Contributions}

AT and AG wrote the manuscript. AT, AG, AC, GA, PC, MG, MD'A, GDM took care of the patient. All authors approved the manuscript.

\section{References}

1. Olivieri NF (1999) The $\beta$-thalassemias. N Engl J Med 341: 99-109.

2. Taher AT, Weatherall DJ, Cappellini MD (2017) Thalassemia. Lancet 391: 155-167.

3. Cappellini MD, Musallam KM, Taher AT (2009) Insight onto the pathophysiology and clinical complications of thalassemia intermedia. Hemoglobin 33:S145-59.
4. Taher A, Isma'eel H, Cappellini MD (2006) Thalassemia intermedia: revisited Blood Cells, Molecules, and Diseases 37: 12-20.

5. Eldor A, Rachmilewitz EA (2002) The hypercoagulable state in thalassemia Blood 99: 36-43.

6. Succar J, Musallam KM, Taher AT (2011) Thalassemiaandvenous thromboembolism. Mediterr J Hematol Infect Dis 3: e2011025.

7. Taher AT, Otrock ZK, Uthman I, Cappellini MD (2008) Thalassemia and hypercoagulability. Blood Rev 22: 283-292.

8. Cappellini MD, Musallam KM, Poggiali E, Taher AT (2012) Hypercoagulability in non-transfusion-dependentthalassemia. Blood Rev 1: S20-23.

9. Taher AT, Musallam KM, Karimi M, El-Beshlawy A, Belhoul K, et al. (2010) Splenectomy and thrombosis: the case of thalassemia intermedia. J ThrombHaemost 8: 2152-2158.

10. Cappellini MD, Grespi E, Cassinerio E, Bignamini D, Fiorelli G (2005) Coagulation and splenectomy: an overview. Ann N Y AcadSci 1054: 317-324.

11. Karimi M, Musallam MK, Cappellini MD, Daar S, El-Beshlawy A, et al. (2011) Risk factors for pulmonary hypertension in patients with $\beta$ thalassemia intermedia. Eur J Intern Med 22: 607-610.

12. Fraidenburg DR, Machado RF (2016) Pulmonary hypertension associated with thalassemia syndromes. Ann NY AcadSci 1368:127-139.

13. Taher A, Isma'eel H, Mehio G, Bignamini D, Kattamis A, et al. (2006) Prevalence of thromboembolic events among 8,860 patients with thalassemia major and intermedia in the Mediterranean area and Iran. ThrombHaemost 96: 488-491.

14. Del Principe D, Menichelli A, Di Giulio S, De Matteis W, Cianciulli P, et al. (1993) PADGEM/GMP-140 expression on platelet membranes from homozygous beta thalassaemic patients. $\mathrm{Br} \mathrm{J}$ Haematol 84: 111-117.

15. Ruf A, Pick M, Deutsch V, Patscheke H, Goldfarb A, et al. (1997) In-vivo platele activation correlates with red cell anionic phospholipid exposure in patients with beta-thalassaemia major. $\mathrm{Br} \mathrm{J}$ Haematol 98: 51-56

16. Eldor A, Lellouche F, Goldfarb A, Rachmilewitz EA, Maclouf J (1991) In vivo platelet activation in beta-thalassemia major reflected by increased plateletthromboxane urinary metabolites. Blood 77: 1749-1753.

17. Musallam KM, Taher AT (2011) Thrombosis in thalassemia: why are we so concerned? Hemoglobin 35: 503-510.

18. Cappellini MD, Poggiali E, Taher AT, Musallam KM (2012) Hypercoagulability in $\beta$-thalassemia: a status quo. Expert Rev Hematol 5: 505-512.

19. Hoeper MM, Bogaard HJ, Condliffe R, Frantz R, Khanna D, et al. (2013) Definitions and diagnosis of pulmonary hypertension. J Am Coll Cardiol 62: D42-D50.

20. Simonneau G, Gatzoulis MA, Adatia I, Celermajer D, Denton C, et al. (2013) Updated clinical classification of pulmonary hypertension. J Am Coll Cardiol 62: D34-41.

21. Ussavarungsi K, Burger CD (2014) Pulmonary arterial hypertension in a patients with thalassemia intermedia and reversal with infusion epoprosteno then transition to oral calcium blocker therapy: review of the literature. Pulm Circ 4: 520-526.

22. Galiè N, Humbert M, Vachiery JL, Gibbs S, Lang I, et al. (2016) 2015 ESC ERS Guidelines for the diagnosis and treatment of pulmonary hypertension The Joint Task Force for the Diagnosis and Treatment of Pulmonary Hypertension of the European Society of Cardiology (ESC) and the European Respiratory Society (ERS): Endorsed by: Association for European Paediatric and Congenital Cardiology (AEPC), International Society for Heart and Lung Transplantation (ISHLT). Eur Heart J 37: 67-119.

23. Derchi G, Galanello R, Bina P, Cappellini MD, Piga A, et al. (2014) Prevalence and risk factors for pulmonary arterial hypertension in a large group of $\beta$-thalassemia patients using right heart catheterization: a Webthal study. Circulation 129: 338-345

24. Aessopos A, Farmakis D, Hatziliami A, Fragodimitri C, Karabatsos F, et al (2004) Cardiac status in well-treated patients with thalassemia major. Eur $\mathrm{J}$ Haematol 73: 359-366.

25. Atichartakarn V, Chuncharunee $S$, Chandanamattha $P$, Likittanasombat K, Aryurachai K (2016) Correction of hypercoagulability and amelioration of pulmonary arterial hypertension by chronic blood transfusion in an a splenich hemoglobin E/beta-thalassemia patient. Blood 103: 2844-2846. 
Citation: Tufano A, Gianno A, Coppola A, Esposito R, Conca P, et al. (2018) Recurrent Pulmonary Embolism and Pulmonary Hypertension in a Patient with $\beta$-Thalassemia Intermedia. J Blood Lymph 8: 205. doi:10.4172/2165-7831.1000205

Page 4 of 4

26. Ghofrani HA, D’Armini AM, Grimminger F, Hoeper MM, Jansa P, et al. (2013) Riociguat for the treatment of chronic thromboembolic pulmonary hypertension. N Engl J Med 369: 319-329.

27. Ghofrani HA, Galiè N, Grimminger F, Grünig E, Humbert M, et al. (2013) Riociguat for the treatment of pulmonary arterial hypertension. $\mathrm{N}$ Engl J Med 369: 330-340.
28. Tam DH, Farber HW (2006) Pulmonary hypertension and beta-thalassemia major: report of a case, its treatment, and a review of the literature. American Journal of Hematology 81: 443-447.

29. Chueamuangphan N, Wongtheptian W, Patumanond J, Sukonthasarn A Chuncharunee S, et al. (2014) Effect of acetylsalicylic acid on thalassemia with pulmonary arterial hypertension. Int J Gen Med 7: 525-529. 